\title{
Survivability of Tether throughout Deorbiting
}

\author{
By Hiroshi HIRAYAMA ${ }^{1)}$, Ieyoung $\mathrm{KIM}^{1)}$ and Toshiya HANADA ${ }^{1)}$ \\ ${ }^{1)}$ Department of Aeronautics and Astronautics, Kyushu University, Fukuoka, Japan
}

(Received July 8th, 2009)

Electrodynamic tether is a promising candidate device to remove space debris from low earth orbit. It can decelerate space debris without propellant until reentry to atmosphere. An argument of the tether is that it can be easily cut by impacts with relatively small particles. Double lined tether is proposed to improve survivability. This paper describes a method to estimate survivability of the double tether. First, fatality rates are formulated, which means how often the tether will be cut. Second, degradation of the double tether is modeled as a stochastic process. Finally, possibilities to survive from initial altitudes to atmospheric reentry can be evaluated. Result shows that the single tether is hopeless to survive though; the double tether can survive with adequate design parameters. This study gives guidelines to design parameters such as diameter, clearance and number of segments of the double tether.

Key Words: Debris, Tether, Deorbit

\section{Nomenclature}

$\begin{array}{ccl}A & : & \text { effective cross sectional area } \\ D & : & \text { diameter of debris } \\ D_{T} & : & \text { diameter of tether } \\ D_{T C} & : & \text { critical diameter of tether } \\ H & : & \text { altitude } \\ h & : & \text { clearance between strands } \\ L & : & \text { length of tether } \\ L_{S} & : & \text { length of loop } \\ N & : & \text { number of loops } \\ n & : & \text { number of altitude ranges } \\ R_{F} & : & \text { fatality rate } \\ X_{i} & : & \text { probability of } i \text { strands survive } \\ t & : & \text { time in year } \\ \phi & : & \text { differential flux } \\ \Phi & : & \text { cumulative flux } \\ \theta & : & \text { impact azimuth } \\ \theta_{C} & : & \text { critical impact azimuth } \\ \text { Subscripts } & \\ i j & : \text { transition from } i \text { to } j \text { strand(s) survive }\end{array}$

\section{Introduction}

Space tethers will enable various space missions in technologies, transportations and sciences. The tether with end mass provides passive attitude stability with gravity gradient toque. Tumbling motion of tethered satellites can improve efficiency of orbit transfer maneuver.

Electrodynamic tethers (EDT) are electrically conductive tethers with electron emitter at its end as illustrated in Fig. 1. EDT is extended vertically and flies through geomagnetic field. It induces voltage and current along the conductive tether. The current in geomagnetic field generates Lorentz force to accelerate or decelerate the space craft substantially without propellant. Promising application of EDT is to remove abandoned satellites from low earth orbit (LEO) by decelerating until atmospheric reentry.
An argument of using space tether is reliability of such a thin and long tether. The tether can easily be cut by relatively small meteoroids and orbital debris, in addition expected collision rate is considerably large because of its wide effective cross section. Hoyt et al. ${ }^{1)}$ proposed a realistic design of Terminator Tether ${ }^{\mathrm{TM}}$ for deorbiting mission, which uses multi line tethers to improve the survivability. In order to investigate this problem, Inter-Agency Space Debris Coordination Committee (IADC) raised an action item to estimate possibilities of survival of EDTs until deorbiting mission success. IADC members of three countries participated in the study: Institute of Information Science and Technologies, National Research Council (ISTI/CNR), on behalf of the Italian Space Agency (ASI); Kyushu University (KU) on behalf of the Japan Aerospace Exploration Agency (JAXA) and Johnson Space Center (JSC) of the American National Aeronautics and Space Administration (NASA). ${ }^{2-6}$

Past works in KU were concerned to the double lined tether. Oishi ${ }^{2)}$ formulated effective cross-sectional areas and fatality rates of the double tether. And then Hirayama ${ }^{5)}$ introduced probabilistic state variables to explain degradation of the double tether as a stochastic process in which survivabilities are formulated in differential equation of state variables. It was applied to evaluate survivability of deorbiting mission ${ }^{2)}$ and their own project for tether experiment satellite. ${ }^{5)}$ The results showed lower survivability than that of ISTI/CNR because the Italian method does not take account of possibility to be severed both strands by a single impact.

When dealing with deorbiting, numerical calculations are divided in some altitude ranges because orbital environments depend on altitude. The earlier method of KU still overestimated the survivability throughout the deorbiting. It was calculated as a sum of products of survivabilities in each altitude ranges. So the state of degradation of the double tether was not transferred correctly to next altitude range. This problem was fixed by Hirayama ${ }^{6}$ to conjugate state variables correctly from initial altitude to atmospheric reentry.

This paper shows numerical evaluations of survivability of the double tether throughout deorbiting using latest method. It gives guideline to design parameters for the tethers. 


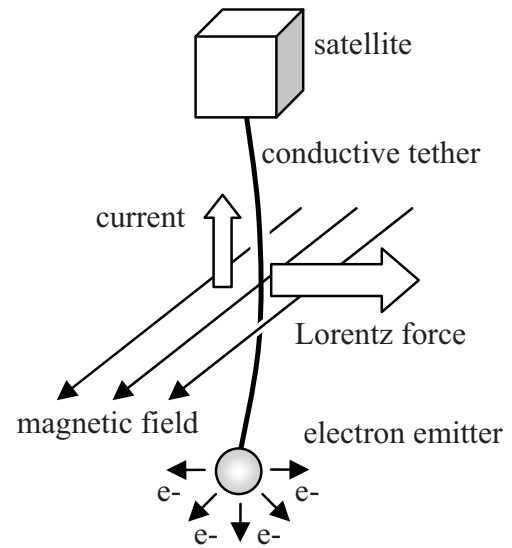

Fig. 1. Schematic of electrodynamic tether.

\section{Environmental Model}

Environment of space debris in certain orbit is expressed in form of cumulative flux which means expected number of annual impacts by debris larger than $D$ in diameter on a sphere of $1 \mathrm{~m}^{2}$ in cross section. Differential flux is also used to express flux in certain diameter, which is derivative of the cumulative flux as defined in Eq. (1).

$$
\phi(D)=-\frac{d}{d D} \Phi(D)
$$

MASTER-2005 by ESA and ORDEM2000 by NASA are popular numerical models. MASTER is based on historical events producing objects and theoretical propagation. On the other hand ORDEM depends on observation data from ground based radars, telescopes and surface inspections on retrieved specimens from space. Fig. 2 shows cumulative fluxes on equatorial circular orbit of $800 \mathrm{~km}$ in altitude, calculated by MASTER and ORDEM. They well match in larger objects, but differ at worst ten times around $1 \mathrm{~mm}$ in diameter because of lack of true knowledge about that region.

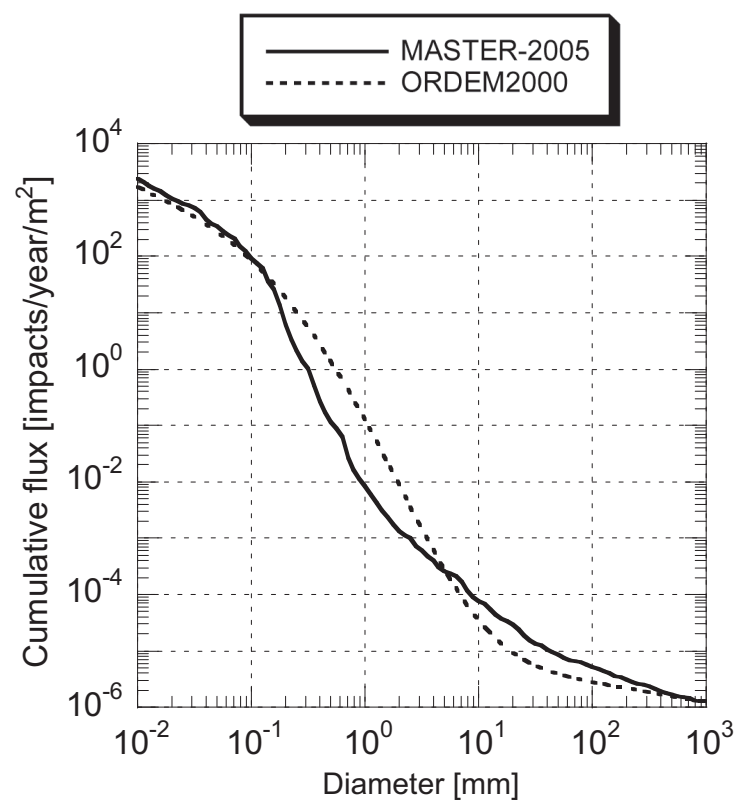

Fig. 2. Cumulative flux. (altitude $=800 \mathrm{~km}$, inclination $=0$, year $=2005$ )

\section{Fatality Rate}

Fatality rates stand for annual expected number of severing the tether. They correspond to time constants of exponentially decaying survivability. A method to evaluate the fatality rates is explained in this section.

First, calculate effective cross-sectional area, where incoming debris can sever one or two of the tethers. In case of the double tether, the cross-sectional area is averaged around incident azimuth of the debris. Then the fatal impact rates are calculated by integrating a product of the effective crosssectional area and differential flux of debris with respect to a diameter of the debris.

\subsection{Single tether}

Shape of a conventional single strand tether is modeled as a long cylinder as shown in Fig. 3. Severing criterion is geometrically assumed that the tether should be severed if a spherical debris larger than $D_{C}$ collides and edge of the debris passes within $D_{T C} / 2$ from a symmetric axis of the tether. We chose parameters $D_{C}$ and $D_{T C}$ as follows. ${ }^{7}$

$$
\begin{gathered}
D_{C}=D_{T} / 3 \\
D_{T C}=0.7 D_{T}
\end{gathered}
$$

Considering that most debris come from horizontally and the tether is vertically stabilized, they can be assumed to intersect perpendicularly. The effective cross-sectional area for the debris of $D$ in diameter becomes

$$
A(D)=L\left(D_{T C}+D\right)
$$

The fatal impact rate of the single tether is obtained by integrating product of the cross-sectional area and the differential flux from lower critical to infinite diameter.

$$
\begin{aligned}
R_{F} & =\int_{D_{C}}^{\infty} A(D) \phi(D) d D \\
& =L\left\{D_{T C} \Phi\left(D_{C}\right)+\int_{D_{C}}^{\infty} D \phi(D) d D\right\}
\end{aligned}
$$

Since first term is dominant in Eq. (5) in general, $\Phi\left(D_{C}\right)$ affects strongly to the fatal impact rate.

The survivability of the single tether after exposed time $t$ in year decays exponentially with a time constant equal to inverse of the fatality rate.

$$
X(t)=X(0) \exp \left[-R_{F} t\right]
$$

where $X(0)$ is initial value of the survivability, obviously 1 at a beginning of the mission.

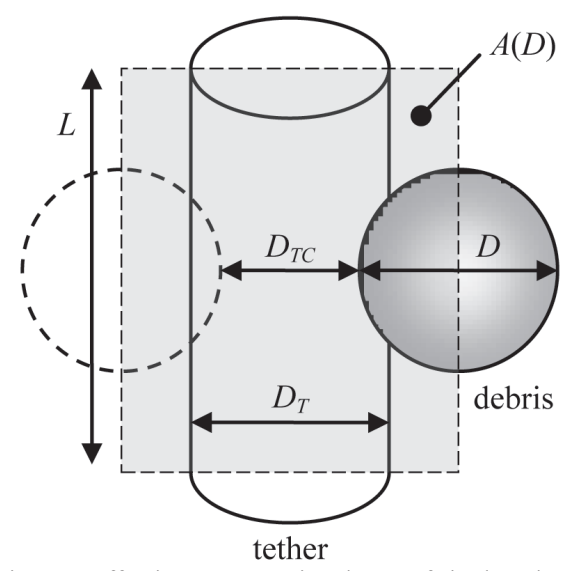

Fig. 3. Effective cross-sectional area of single tether. 


\subsection{Double tether}

Double tether is modeled as shown in Fig. 4, a pair of parallel tethers are knotted every certain distance. Both strands are placed to keep clearance between them. The clearance is given by small difference of length of the parallel strands and elastic behavior of them. Therefore we use $h$ as an effective value because the clearance is not strictly controlled and differs along the tether.

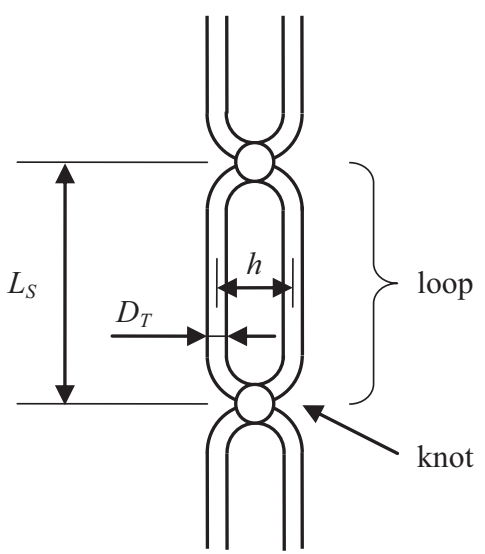

Fig. 4. Model of double tether

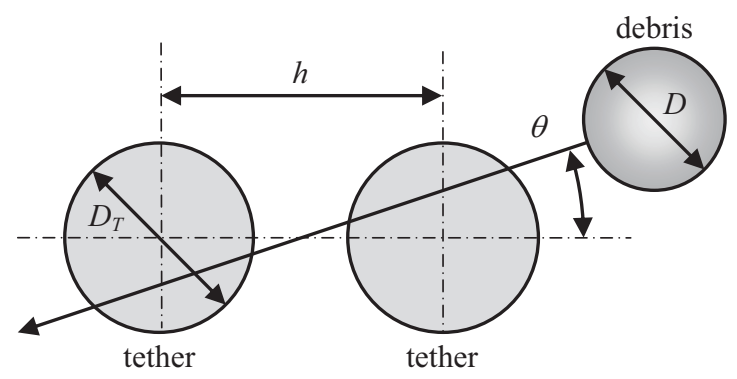

Fig. 5. Geometry of collision to double tether (section view)

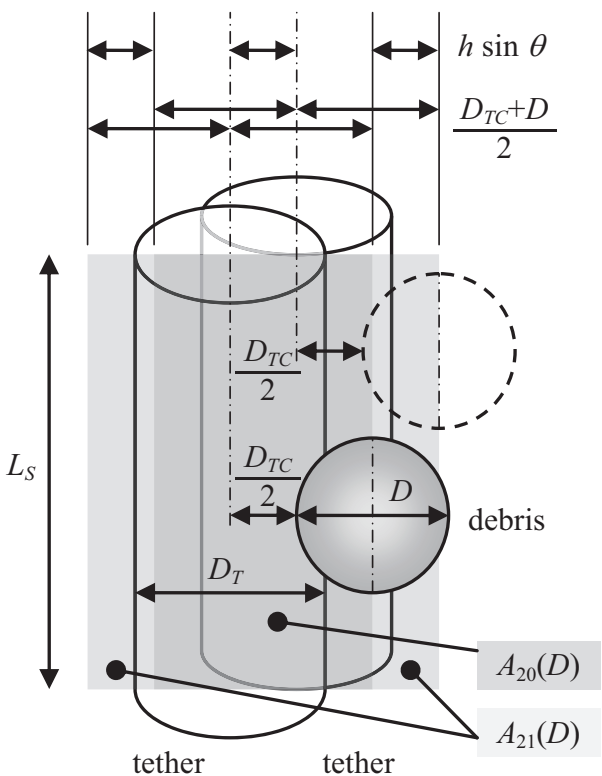

Fig. 6. Effective cross-sectional areas of double tether. (viewed from incident direction)
Entire tether is segmented into $N$ loops. Number of knots along the tether is $N-1$. If length of the knot is smaller enough than that of the loop, we can neglect the size the knot. The length of each loop is

$$
L_{S}=L / N
$$

Double tether is severed in three possible cases.

(1) a knot is severed by a single impact

(2) both strands in a same loop are severed together by a single impact

(3) both strands in a same loop are severed independently by two impacts

Probability of the case (1) is small enough to neglect according to the former work. ${ }^{6}$ Effective cross-sectional areas in case (2) and (3) depend on the in-coming azimuth of orbital debris and distance between the two strands must be accounted for, as shown in Fig. 5 and Fig. 6. Orbital debris greater than $h-D_{T C}$ have possibility to sever both strands simultaneously with a single impact, regardless of its in-coming azimuth. The cross-sectional area for severing both strands with the single impact is

$$
\begin{aligned}
A_{20}(D) & =\frac{2}{\pi} \int_{0}^{\pi / 2} L_{S}\left(D_{T C}+D-h \sin \theta\right) d \theta \\
& =L_{S}\left(D_{T C}+D-\frac{2}{\pi} h\right)
\end{aligned}
$$

If the diameter of the debris is smaller than $h-D_{T C}$, it can sever the both strands simultaneously only in the angle range

$$
0 \leq \theta \leq \sin ^{-1} \frac{D_{T C}+D}{h}\left(\equiv \theta_{C}\right)
$$

Therefore the cross-sectional area for such small debris is

$$
\begin{aligned}
\mathrm{A}_{20}(D) & =\frac{2}{\pi} \int_{0}^{\theta_{C}} L_{S}\left(D_{T C}+D-h \sin \theta\right) d \theta \\
& =\frac{2}{\pi} L_{S}\left[\left(D_{T C}+D\right) \theta_{C}-h\left(1-\cos \theta_{C}\right)\right]
\end{aligned}
$$

The effective cross-sectional area for severing only one strand of the loop with the single impact can be obtained in the same way mentioned above. When the orbital debris is larger than $h-D_{T C}$, the effective cross-sectional area for severing only one strand of the loop with the single impact can be obtained as follows

$$
A_{21}(D)=\frac{2}{\pi} \int_{0}^{\pi / 2} 2 L_{S} h \sin \theta d \theta=\frac{4}{\pi} L_{S} h
$$

When the orbital debris is smaller than $h-D_{T C}$ $A_{21}(D)$

$$
\begin{aligned}
& =\frac{2}{\pi}\left[\int_{0}^{\theta_{C}} 2 L_{S} h \sin \theta d \theta+\int_{\theta_{C}}^{\pi / 2} 2 L_{S}\left(D_{T C}+D\right) d \theta\right] \\
& =\frac{4}{\pi} L_{S}\left[\left(D_{T C}+D\right)\left(\frac{\pi}{2}-\theta_{C}\right)+h\left(1-\cos \theta_{C}\right)\right]
\end{aligned}
$$

The fatal impact rate for severing two strands of the same loop simultaneously is given by

$$
R_{F 20}=\int_{D_{C}}^{\infty} A_{20}(D) \phi(D) d D
$$

and that for severing only one strand of the loop is given by

$$
R_{F 21}=\int_{D_{C}}^{\infty} A_{21}(D) \phi(D) d D
$$

Taking account for the relation that

$$
A_{20}(D)=A_{10}(D)-A_{21}(D) / 2
$$


where $A_{10}(D)$ is the effective cross sectional area of one strand, identical to a single tether of length $L_{S}$, we can find equations

$$
\begin{aligned}
& R_{F 20}=R_{F 10}-R_{F 21} / 2 \\
& R_{F 21}=2\left(R_{F 10}-R_{F 20}\right)
\end{aligned}
$$

where $R_{10}$ is the fatal impact rate of the single tether of length $L_{S}$. Therefore, we have to integrate only one of Eq. (13) or (14) to evaluate $R_{F 20}$ or $R_{F 21}$, and then the other is easily obtained from Eq. (16) or (17). When we increase clearance $h / D_{T}$, those fatality rates approach

$$
\begin{gathered}
\lim _{h / D_{T \rightarrow \infty}} R_{F 20}=0 \\
\lim _{h / D_{T \rightarrow \infty}} R_{F 21}=2 R_{F 10}
\end{gathered}
$$

In order to avoid numerical error, using Eq. (17) is better than (16) in case of wide clearance.

\section{Survivability}

\subsection{Stochastic process of damaging}

Since damaging of the double tether should be treated as a stochastic process, the probabilistic state variables $X_{i}(t)$ were introduced to explain the degradation. ${ }^{5)}$ The subscript $i$ represents the number of strands surviving in a tether loop as shown in Fig. 7. $X_{i}(t)$ gives the probability of $i$ strand(s) is/are surviving after a certain exposed time. It is clear that

$$
X_{2}(t)+X_{1}(t)+X_{0}(t)=1
$$

Initial values of undamaged tether should be

$$
X_{2}(0)=1, X_{1}(0)=0, X_{0}(0)=0
$$

at the beginning of the mission.

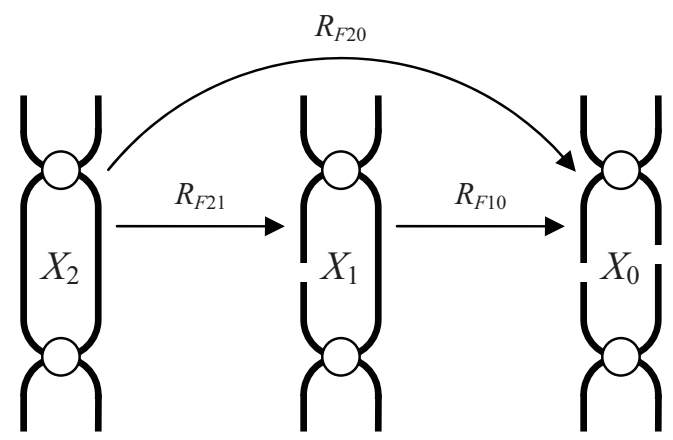

Fig. 7. Stochastic state transition as degrading double tether.

Annual transition rate from state $X_{i}$ to $X_{j}$ is equal to the fatality rate $R_{F i j}$, therefore behavior of the probabilistic state variables can be described as a set of simultaneous differential equations.

$$
\left\{\begin{array}{l}
\dot{X}_{2}(t)=-\left(R_{F 20}+R_{F 21}\right) X_{2}(t) \\
\dot{X}_{1}(t)=R_{F 21} X_{2}(t)-R_{F 10} X_{1}(t)
\end{array}\right.
$$

These equations can be solved to give

$$
\begin{aligned}
& X_{2}(t)=X_{2}(0) \exp \left[-\left(R_{F 20}+R_{F 21}\right) t\right] \\
& X_{1}(t)= \\
& {\left[X_{1}(0)+X_{2}(0) \frac{R_{F 21}\left(1-\exp \left[-R_{X} t\right]\right)}{R_{X}}\right] \exp \left[-R_{F 10} t\right]} \\
& \text { where } R_{X}=R_{F 20}+R_{F 21}-R_{F 10}
\end{aligned}
$$

Remembering the relation of the fatality rates in Eq. (16), the solution Eq. (23) and (24) can be simplified to

$$
\begin{aligned}
& X_{2}(t)=X_{2}(0) \exp \left[-\left(\frac{R_{F 21}}{2}+R_{F 10}\right) t\right] \\
& X_{1}(t)= \\
& {\left[X_{1}(0)+2 X_{2}(0)\left(1-\exp \left[-\frac{R_{F 21}}{2} t\right]\right)\right] \exp \left[-R_{F 10} t\right]}
\end{aligned}
$$

And then, the probability of at least one strand in the loop have survived after a certain time is

$$
\begin{aligned}
& X_{2}(t)+X_{1}(t)= \\
& {\left[X_{1}(0)+X_{2}(0)\left(2-\exp \left[-\frac{R_{F 21}}{2} t\right]\right)\right] \exp \left[-R_{F 10} t\right]}
\end{aligned}
$$

Finally probability of all $N$ loops along whole tether have survived is given by

$$
\left(X_{2}(t)+X_{1}(t)\right)^{N}
$$

\subsection{Probability of successful reentry}

Considering deorbiting from the higher orbit into atmospheric reentry, fatality rates are not constant values as formulated in section 3 , but should be functions of altitude. However, updating the fatality rate requires much calculation time, and analytic formulation in section 4.1 cannot be used.

Instead of treating them as continuous functions, we divided altitude into some ranges and assumed fatality rates are constant within each range. When the initial altitude is $H_{0}$ and atmospheric reentry altitude is $H_{a}$, we divide the altitude range into $n$ small ranges

$$
\left\{\begin{array}{l}
H_{0} \rightarrow H_{1} \\
H_{1} \rightarrow H_{2} \\
\vdots \\
H_{i-1} \rightarrow H_{i} \\
\vdots \\
H_{n-1} \rightarrow H_{n}\left(=H_{a}\right)
\end{array}\right.
$$

Elapsed times to decay through $i$-th range are $t_{i}$, they are given from electrodynamic and aerodynamic analysis. The fatality rates in $i$-th range are calculated in middle altitude.

$$
\left(H_{i-1}-H_{i}\right) / 2
$$

Final values of solution (25) and (26) in each altitude ranges should be cascaded to initial values of next ranges.

$$
\left\{\begin{array}{l}
X_{2}(0)\left[H_{i} \rightarrow H_{i+1}\right]=X_{2}\left(t_{i}\right)\left[H_{i-1} \rightarrow H_{i}\right] \\
X_{1}(0)\left[H_{i} \rightarrow H_{i+1}\right]=X_{1}\left(t_{i}\right)\left[H_{i-1} \rightarrow H_{i}\right]
\end{array}\right.
$$

Finally, value (32) of lowest altitude gives probability of survival until atmospheric reentry.

$$
\left(X_{2}\left(t_{n}\right)+X_{1}\left(t_{n}\right)\right)^{N}\left[H_{n-1} \rightarrow H_{n}\right]
$$

\section{Simulation Model}

Investigations of the survivability were performed based on the design of the Terminator TetherTM. ${ }^{1)}$ Parameters for the calculations are listed in Table 1. Indeed the final altitude of $250 \mathrm{~km}$ seems still higher than reentry altitude, where is required goal for EDT to survive. Satellite can decay quickly after this altitude by aerodynamic drag even if the EDT fails. Altitude ranges were divided into every $100 \mathrm{~km}$ for calculation as explained in section 4.2., except for lowest one is from 400 to $250 \mathrm{~km}$. Elapsed times for deorbit from various initial orbits are shown in Fig. 8. ${ }^{1)}$ The lower inclination gives shorter elapsed time because EDT can generate larger deceleration force in lower inclination. 
Table 1. Parameters for investigation.

\begin{tabular}{|c|c|}
\hline Parameters & Values \\
\hline \hline Length of tether $L$ & $7.5 \mathrm{~km}$ \\
\hline $\begin{array}{c}\text { Number of loops } N \\
\left(\text { Length of loops } L_{S}\right)\end{array}$ & 75,750 \\
& $(100,10 \mathrm{~m})$ \\
\hline Diameter of tether $D_{T}$ & $0.5,1.0 \mathrm{~mm}$ \\
\hline Clearance of tether $h$ & $10,100 \mathrm{~mm}$ \\
\hline Initial altitude $H_{0}$ & $400,500, \ldots, 1400 \mathrm{~km}$ \\
\hline Final altitude $H_{a}$ & $250 \mathrm{~km}$ \\
\hline Orbital inclination & $0,25,50,75$ degrees \\
\hline Environmental model & MASTER-2005, ORDEM2000 \\
\hline
\end{tabular}

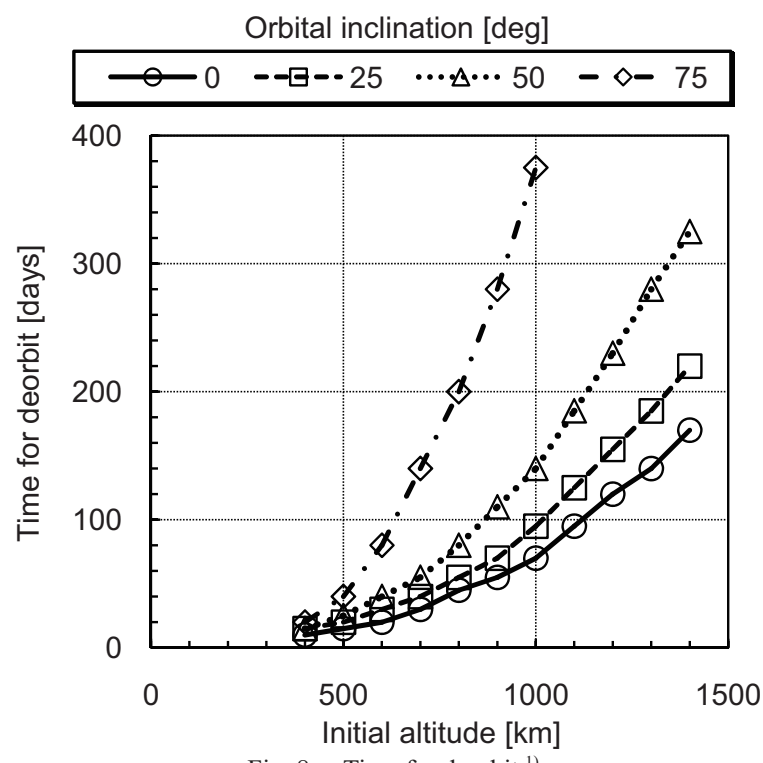

Fig. 8. Time for deorbit

\section{Results}

At first survivabilities of the diameter and the clearance of the tethers were evaluated along with that of single tethers. Diameters of tethers are 0.5 and $1.0 \mathrm{~mm}$, clearances are 10 and $100 \mathrm{~mm}$. Other conditions are chosen to give best results, i.e. $N=750$, inclination is 0 , MASTER-2005. Fig. 9 shows substantial advantage of using the double tethers. Thinner double tethers have more possibility then the thicker single tether. Single tethers are hopeless to survive even in the easiest condition.

We expect EDTs to survive more than $90 \%$ from initial altitude of $800-1000 \mathrm{~km}$ where is crowded with abandoned satellites to be removed by EDTs. From this requirement we consider only the double tethers of $1.0 \mathrm{~mm}$ in diameter for further investigations.

And then effect of number of loops and clearances are shown in Fig. 10. The more number of loops and the wider clearance give more survivability.

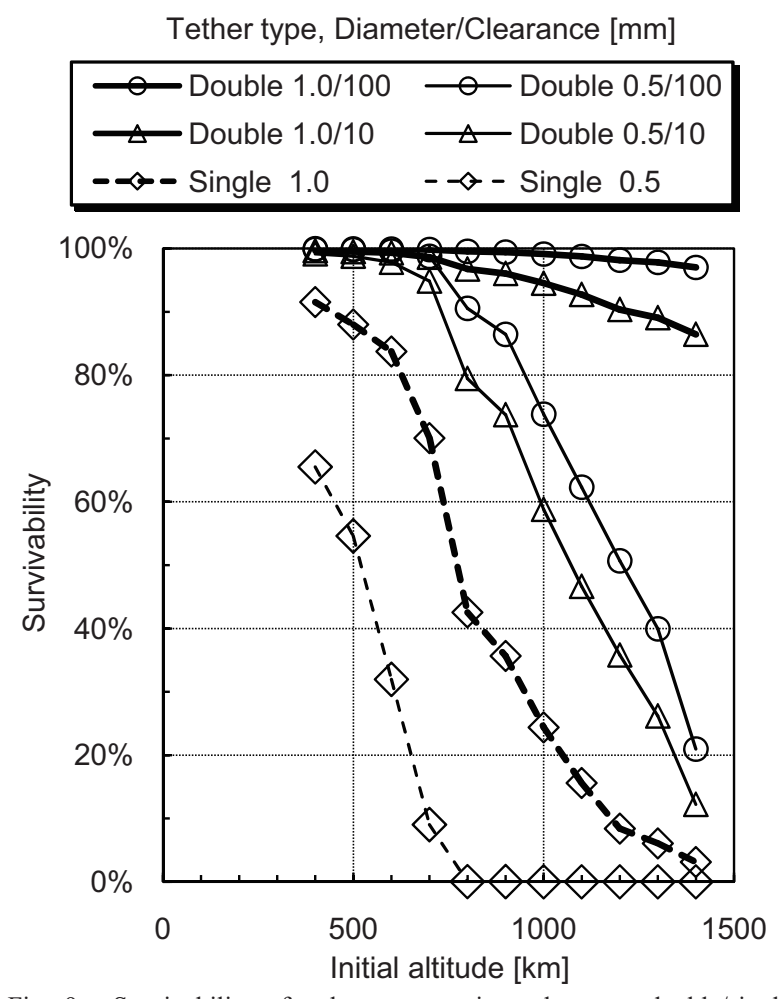

Fig. 9. Survivability of tethers, comparisons between double/single, diameters and clearances. (MASTER-2005, inclination $=0 \mathrm{deg}, N=750$ )

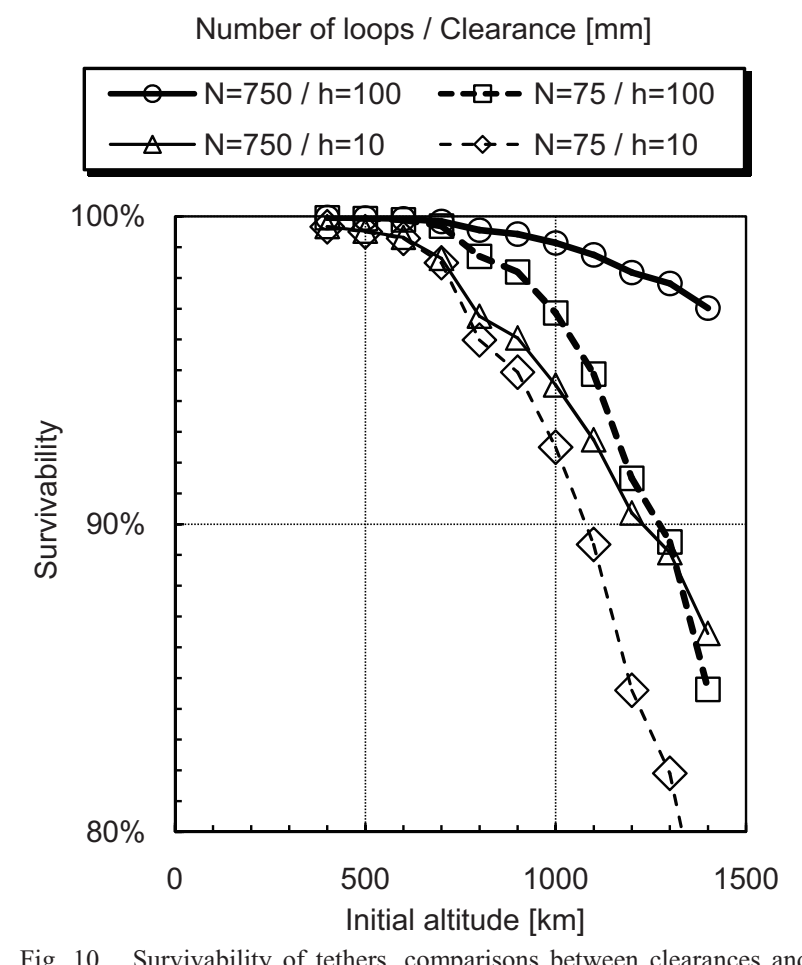

Fig. 10. Survivability of tethers, comparisons between clearances and numbers of loops. (MASTER-2005, inclination $=0 \mathrm{deg}, D_{T}=1.0 \mathrm{~mm}$ ) 


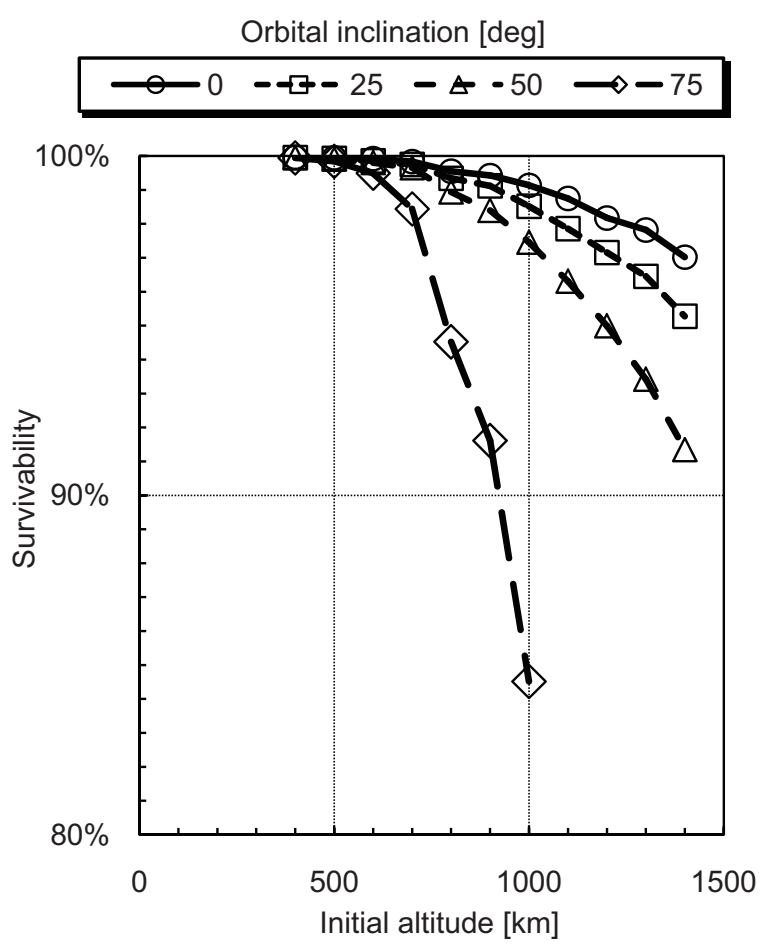

Fig. 11. Survivability of tethers, comparisons between inclinations (MASTER-2005, $D_{T}=1.0 \mathrm{~mm}, h=100 \mathrm{~mm}, N=750$ )

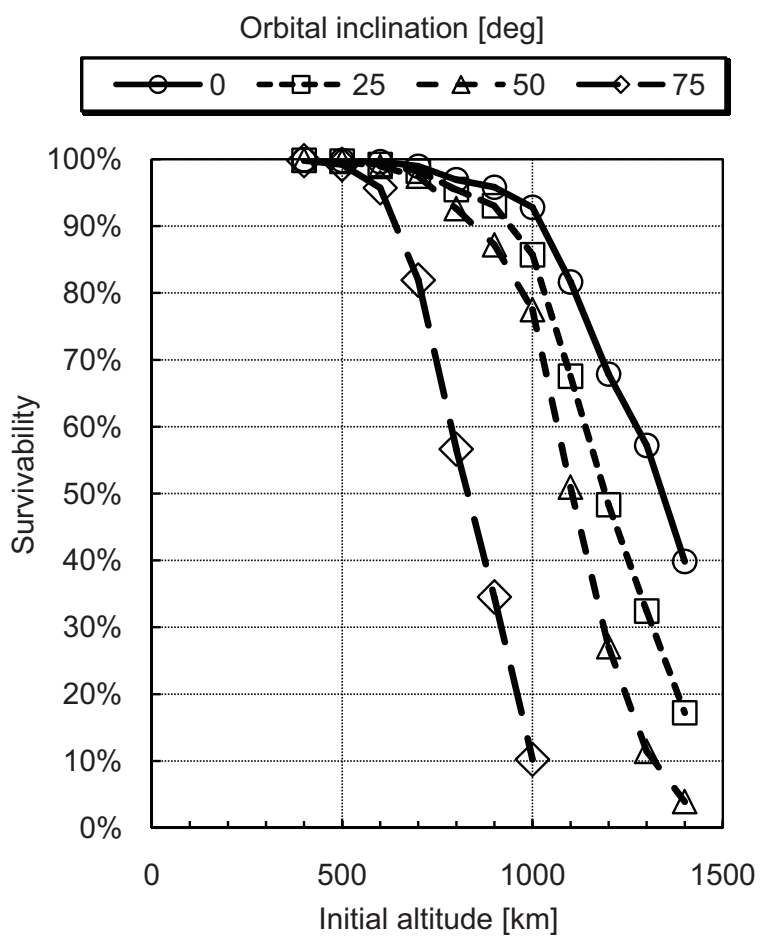

Fig. 12. Survivability of tethers, comparisons between inclinations. (ORDEM2000, $D_{T}=1.0 \mathrm{~mm}, h=100 \mathrm{~mm}, N=750$ )
At last, the double tether of better design parameters, $D_{T}=1.0 \mathrm{~mm}, h=100 \mathrm{~mm}, N=750\left(L_{S}=10 \mathrm{~m}\right)$, was investigated in different orbital inclinations, $0,25,50$ and 75 degrees. Differences between environmental models are also compared. Fig. 11 shows results using MASTER-2005, and Fig. 12 using ORDEM2000. Deorbiting from the higher inclinations result in lower possibility to survive because of longer elapsed time. Results from ORDEM2000 are much lower than that from MASTER-2005. The reason is that fatality rates are sensitive to the cumulative flux for critical diameter $\Phi\left(D_{C}\right)$, where ORDEM2000 gives higher value around $1 \mathrm{~mm}$ region as shown in Fig. 2. There is no clue which model is more realistic in this region. If we believe ORDEM2000, the tethers must be designed thicker to achieve sufficient possibility to survive throughout deorbiting.

\section{Conclusion}

The method to estimate the survivability of the EDT throughout the deorbiting mission was explained. It is based on geometry of the tethers and flux data from environmental model of orbital debris and meteoroids.

According to the scenario of the Terminator Tether ${ }^{\mathrm{TM}}$, the survivabilities were evaluated and compared in certain range of design parameters. The results show that the double tethers whose $D_{T}>1.0 \mathrm{~mm}, h>100 \mathrm{~mm}$ and $N>750$ have enough survivability on the environment model of MASTER-2005.

Although the thicker tether has higher survivability, it causes increase in mass. Diameter of the tether should be designed after trade-off between survivability and mass.

\section{References}

1) Hoyt, R. P. and Forward R. L.: Performance of the Terminator Tether $^{\mathrm{TM}}$ for Autonomous Deorbit of LEO Spacecraft, 35th AIAA/ASME/SAE/ASEE Joint Propulsion Conference \& Exhibit, Paper AIAA 99-2839, 1999

2) Oishi, A., Hirayama, H., Hanada, T., Yasaka, T., Pardini, C. and Anselmo, L.: Assessment of Collision Risk to Electrodynamic Tether Used for De-Orbiting, 55th International Astronautical Congress, IAC-04-IAA.5.12.5.09, Vancouver, 2004.

3) Pardini, C., Anselmo, L., Hanada, T. and Hirayama, H.: Assessing the Vulnerability to Debris Impacts of Electrodynamic Tethers During Typical De-orbiting Missions, Proceedings of the 4th European Conference on Space Debris, Darmstadt, Germany, 2005, Paper SPACE2005-A-00009, pp. 353-360.

4) Pardini, C., Hanada, T., Krisko, P. H., Anselmo, L. and Hirayama, H.: Are de-Orbiting Missions Possible using Electrodynamic Tethers? Task Review from the Space Debris Perspective, Acta Astronautica, 60 (2007), pp. 916-929.

5) Hirayama, H., Oishi, A., Hanada, T. and Yasaka, T.: Stochastic Analysis of Survivability of Double Tether, Acta Astronautica, 62 (2008), pp. 54-58

6) Hirayama, H., Oishi, A. and Hanada, T.: Survivability of Electrodynamic Tether until Atmospheric Reentry, ISTS 2006-r-2-18, Kanazawa, 2006.

7) Cosmo, M. L. and Lorenzini, E. C.: Tethers in Space Handbook, 3rd ed., Smithsonian Astrophysical Observatory, Cambridge, 1997, pp. 180-181. 